\title{
Large Eddy Simulation of Spanwise Rotating Turbulent Channel Flow with Subgrid-Scale Eddy Viscosity Model Based on Helicity
}

\author{
Zhou Jiang $^{1, *}$ and Changping $\mathrm{Yu}^{2}$ \\ ${ }^{1}$ College of Aerospace Engineering, Chongqing University, Chongqing, China \\ ${ }^{2}$ LHD, Institute of Mechanics, Chinese Academy of Sciences, 100190, Beijing, China
}

Received 18 August 2018; Accepted (in revised version) 6 December 2018

\begin{abstract}
Fully developed rotating turbulent channel flow (RTCF) has been numerically investigated using large-eddy simulation (LES). The subgrid-scale (SGS) eddy viscosity model is base on an SGS helicity dissipation balance and the spectral relative helicity. Posterior test has been implemented to an RTCF with rotation in spanwise direction. The friction Reynolds number $R e_{\tau}=u_{\tau} \delta / v$ based on wall shear velocity $u_{\tau}$, half width of the channel $\delta$ and the kinematic viscosity $v$ is 180. Two rotation numbers $R o_{\tau}=2 \Omega \delta / u_{\tau}$ equal to 22 and 80 have been computed with respective grid resolution. The results from dynamic Smagorinsky model (DSM) and direct numerical simulation (DNS) are used as references. The results demonstrate that the eddy viscosity model can predict both the precise velocity profile and the turbulent intensity.
\end{abstract}

AMS subject classifications: 65M10, 78A48

Key words: Large eddy simulation, rotating turbulent channel flow, turbulence.

\section{Introduction}

Computational fluid dynamics (CFD) is an important method in turbulence prediction and has been rapidly developed in recent decades. Benefited from the booming of the performance of supercomputers, the LES approach gradually plays an increasingly important role in CFD, especially in unsteady flows and massive separated flows [1-5]. In LES, the physical quantities are divided into the resolved-scale and subgrid-scale (SGS) sections by means of low-pass filtering. Large scale structure is simulated directly, while the small scale structure need to be modeled by the SGS stress model.

*Corresponding author.

Email: jiangzhou@cqu.edu.cn (Z. Jiang) 
Although LES approaches have made great progresses in many predictions of engineering turbulent flows, the implementations in fully developed rotating turbulent channel flow (RTCF) are not plentiful. In spanwise RTCF, the Coriolis force is perpendicular to the mean velocity vector and is parallel to the wall normal direction. Since the direction of mean velocity and the rotation is fixed, the direction of Coriolis force points from one wall to the other wall, i.e., the Coriolis force presses one wall and sucks another wall. Hence, the mechanism of the two side is different. Under the effect of Coriolis force, the flow field is divided into two parts. In one part of the channel, the flow get stabilizes. However, in the other part of the channel, the flow become destabilizes. The two parts are denoted by suction side and pressure side respectively. Therefore, turbulence is suppressed in the suction side, while enhanced in the pressure side. As the rotation number raises, the suction side will tend to laminarization, consequently the SGS model should be reduced to zero. Hence, the flow pattern of low rotation number is distinguished from that of high rotation number. In the low rotation case, the turbulence behavior is continuous. however, in the high rotation case, since the flow field is divided into two layers, a buffer region of the turbulence quantities exist. In addition, the Coriolis force is proportional to the vorticity. Hence, the vorticity is closely connected to spanwise RTCFs.

Therefore, the SGS modeling of RTCF is a challenge in numerical simulation. The most commonly used method in RTCF is still DNS method at the present time. However, the SGS modeling of RTCF is of great importance in both scientific researches and engineering applications. The most widely used SGS models are base on homogenous turbulence or wall-bounded turbulence and formulated by strain rate tensor or velocity gradient tensor. The relation between SGS model and vorticity has not attracted enough attentions.

In LES, the most commonly used models is eddy viscosity model, among which Smagorinsky model (SM) is most typical due to its robust and simplicity [6]. In most eddy viscosity models, including Vreman's model [7], wall adapting local eddy viscosity model [8], volumetric strain-stretching model [9], the eddy viscosity is a function of resolved velocity gradients. In such models, the influence of vorticity has not been taken account. However, in helical turbulence, the contributions from the helicity have been considered for SGS modeling. Baerenzung et al. [10] extended the eddy-damped quasi normal Markovian approach and introduced a modified spectral eddy-viscosity model for large-eddy simulation in turbulent flows. Yu et al. [12] proposed a refined Smagorinsky model (RSM) based on both velocity gradient and vorticity gradient. The RSM was implemented in simulation of flow past a circular cylinder and the results suggest the helicity is important in SGS modeling. In this paper, the RSM will be implemented in RTCF at $R o_{\tau}=22$ and $R o_{\tau}=80$ to evaluate the performance in such flow case.

The remains of this paper is organized as follows: Section 2 introduces governing equations and SGS models. In Section 3, dynamic RSM is implemented in simulation of RTCF at two rotation numbers. The results are compared with the DNS results and DSM results. Finally, concluding remarks and further discusses are given in Section 4 . 